\title{
Meta
}

Journal des traducteurs

Translators' Journal

\section{La recherche terminologique dans la traduction scientifique et technique japonais-français : une synthèse}

\section{Daniel Gile}

Volume 29, numéro 3, septembre 1984

URI : https://id.erudit.org/iderudit/002902ar

DOI : https://doi.org/10.7202/002902ar

Aller au sommaire du numéro

Éditeur(s)

Les Presses de l'Université de Montréal

ISSN

0026-0452 (imprimé)

1492-1421 (numérique)

Découvrir la revue

Citer cet article

Gile, D. (1984). La recherche terminologique dans la traduction scientifique et technique japonais-français : une synthèse. Meta, 29(3), 285-290.

https://doi.org/10.7202/002902ar d'utilisation que vous pouvez consulter en ligne. 


\section{LA RECHERCHE TERMINOLOGIQUE DANS LA TRADUCTION SCIENTIFIQUE ET TECHNIQUE JAPONAIS-FRANÇAIS : UNE SYNTHÈSE}

Dans l'idéal, pour faire face aux exigences de sa profession, le traducteur scientifique et technique devrait :

- comprendre parfaitement la langue de départ, 
- avoir une connaissance active parfaite de la langue d'arrivée dans les sociolectes pertinents,

- avoir une bonne connaissance des domaines dans lesquels il est spécialisé,

- être familiarisé avec les méthodes de traduction,

- savoir rédiger des textes scientifiques et techniques.

En ce qui concerne les langues de départ et d'arrivée, les méthodes et la compétence rédactionnelle, on peut dire que le talent, la formation et l'expérience permettent de se rapprocher dans une certaine mesure de cet idéal. Par contre, les conditions matérielles de l'exercice de la profession de traducteur et la rapidité de l'évolution des sciences et techniques empêchent, dans la quasi-totalité des cas, la stricte spécialisation dans une matière que l'on connaît bien ${ }^{1}$, et dans l'ensemble, les connaissances spécialisées du traducteur sont très inférieures à celles de l'auteur et des destinataires du texte ${ }^{2}$.

Pour le traducteur, il en résulte parfois des difficultés de compréhension de l'original, et toujours des difficultés dans la restitution du message dans le sociolecte précis des destinataires.

Dans la traduction japonais-français, des contraintes spécifiques s'ajoutent à ces problèmes généraux :

- Si l'on part du principe, généralement admis, que le professionnel traduit vers sa langue maternelle, le travail vers le français doit être fait par des francophones. Or, l'acquisition du japonais présente des difficultés considérables et, à l'heure actuelle, les traducteurs français travaillant à partir du japonais ont un niveau de compréhension de cette langue qui est inférieur au niveau de compréhension confortable qui est souhaitable.

Dans la traduction japonais-français, la condition de parfaite compréhension de la langue de départ n'est donc pas satisfaite.

- Le petit nombre de traducteurs compétents ( 8 personnes seulement dans l'annuaire de la Société française des traducteurs en 1983) exclut d'emblée la spécialisation, et chacun est amené à aborder des textes se situant dans des domaines étrangers à sa formation et à son expérience professionnelle, d'où des lacunes cognitives très marquées.

- La structure linguistique et langagière du japonais se caractérise entre autres par un faible degré d'explicitation ${ }^{3}$. C'est pourquoi le décodage des textes japonais repose davantage sur les connaissances extra-linguistiques que le décodage des textes rédigés dans les langues occidentales 4 .

Il en ressort que l'acquisition d'un bagage cognitif pertinent est plus systématiquement nécessaire dans la traduction japonais-français que dans la traduction entre langues européennes.

Pour les étudiants de $3^{\mathrm{e}}$ année de l'Institut national des langues et civilisations orientales de Paris, dont le niveau de connaissance du japonais est sensiblement infé-

1. Alain Rey (1979) : la Terminologie, noms et notions, Paris, PUF, p. 60.

2. Voir Daniel Moskowitz (1973) : "Le traducteur : récepteur et destinataire du message ", dans ELA, no 12 , octobre-décembre.

3. Voir Shigeo Kawamoto (1976) : Kotoba to kokoro, Tokyo, Iwanamishinsho et Akira Oide (1965) : Nihongo to ronri, Tokyo, Kodanshagendaisho.

4. Voir par exemple Bunkichi Fujimori, "Japon - la langue ", article de l'Encyclopaedia Universalis, et S. Kawamoto, op. cit., p. 251. 
rieur au minimum requis pour la traduction professionnelle, elle est encore plus importante, puisqu'elle doit compenser de grosses lacunes linguistiques.

Dans la pratique, l'acquisition du bagage cognitif pertinent est axée sur la recherche terminologique, dont le point de départ est un terme incompris ou posant des problèmes de restitution dans la langue d'arrivée.

Le présent article décrit et analyse les trois niveaux de la recherche terminologique telle qu'enseignée au cours d'initiation à la traduction scientifique et technique japonais-français à l'INALCO'5.

La présentation est explicative plutôt que directive, et vise à montrer le potentiel et les limites des différents types d'analyse, ainsi que les avantages et les inconvénients des différents types de sources.

Il convient de noter que le terme "recherche terminologique" prend ici un sens plus large que son sens habituel, puisqu'il englobe, outre la recherche terminologique classique axée sur les dictionnaires et autres documents, l'analyse du texte à traduire, et jusqu'à l'analyse du terme inconnu lui-même.

En effet, comme il est expliqué plus loin, les conditions pratiques du travail de traduction japonais-français en France font que les analyses ponctuelles et contextuelles sont souvent plus importantes que la recherche externe.

\section{L'ANALYSE PONCTUELLE}

L'action la plus localisée du traducteur face à un terme difficile est son analyse.

En réalité, la lecture comporte toujours une analyse inconsciente et rapide des formes $^{6}$. L'analyse dont il est question ici, par contre, est une action consciente et délibérée, destinée à résoudre un problème précis.

Dans la traduction des langues occidentales, l'analyse ponctuelle est peu fréquente. Dans la traduction du japonais, elle est rendue nécessaire par l'insuffisance des sources externes, et utile par les caractéristiques lexicales et graphiques du japonais.

Pour analyser un texte inconnu, le traducteur dispose d'indices morphologiques, graphiques et phonétiques, dont l'importance relative varie selon les langues.

En japonais, les liens entre le graphisme et la prononciation sont complexes. Une unité lexicale peut être définie par son graphisme et se prononcer de deux manières différentes. L'unité 薄 板, qui désigne une plaque mince, se prononce "hakuhan" ou “ usuita"; l'unité 赤饭, qui évoque un plat de riz et de haricots rouges, se prononce "sekihan", ou "akameshi". Le mot peut également être défini par sa forme vo-

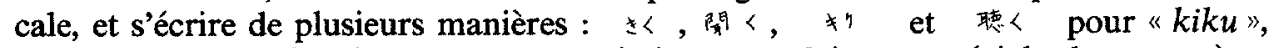
"écouter ". La recherche d'une prononciation secondaire ou spéciale des caractères composant un terme inconnu livre parfois la clef d'un problème, quand la prononciation habituelle ne correspond pas à une entrée dans le dictionnaire.

Indépendamment de leur prononciation, les caractères chinois, que les Japonais ont tendance à juxtaposer en toute liberté pour former de nouveaux mots ${ }^{7}$, sont aussi explicites que les suffixes et préfixes gréco-latins en français, et fournissent à ce titre de précieuses indications sur le sens de l'entité lexicale ainsi créée.

5. Voir Daniel Gile (1983) : «Initiation à la traduction scientifique et technique japonais-français à l'INALCO : la recherche d'une optimisation des méthodes ", Bulletin de l'association des anciens élèves de I'INALCO, juin.

6. Voir François Richaudeau (1973) : le Langage efficace, Paris, Denoël, ou François Richaudeau (1976) : Linguistique pragmatique, Paris, Retz, ou encore (1976), la Lisibilité, du même auteur, chez Retz.

7. Voir Ichiharu Kindaichi (1957) : Nihongo, Tokyo, Iwanamishinsho, p. 25. 
Les katakana, pour leur part, indiquent en général l'origine occidentale d'un mot, et aident par là à orienter les efforts de recherche (il convient de noter à ce propos que le sens japonais d'un terme importé est parfois éloigné de son sens originel ${ }^{8}$ ).

L'analyse ponctuelle, essentiellement graphique et morphologique, des termes japonais inconnus, permet donc d'obtenir de très bons résultats, analogues à ceux que livre l'analyse d'un grand nombre de termes médicaux français et anglais.

\section{L'ANALYSE CONTEXTUELLE}

L'analyse contextuelle, moins localisée que l'analyse ponctuelle, exploite les indices fournis par le texte pour déterminer, ou au moins pour circonscrire le sens du terme problématique. Elle repose sur des éléments cognitifs d'une part, et sur des éléments linguistiques de l'autre, et passe par la recherche de la cohérence et de la vraisemblance, et par l'élimination des contradictions.

\section{L'apport cognitif}

La lecture du texte original dans sa totalité préalablement à la traduction est systématiquement recommandée par les formateurs ${ }^{9}$; elle permet souvent de trouver des explications, des définitions ou des illustrations graphiques de l'objet ou de l'idée que représente le terme problématique, et donne dans tous les cas une vue d'ensemble du message.

En réalité, compte tenu des contraintes pratiques de temps, la lecture intégrale du texte de bout en bout n'est pas toujours possible, ni d'ailleurs indiquée, quand le document en question est volumineux. Une lecture en diagonale, plus appuyée au moment du survol de la table des matières, des têtes de chapitre, de l'index et des illustrations est par contre toujours souhaitable, car elle indique, l'expérience et le flair du traducteur aidant, les passages informatifs susceptibles d'être particulièrement utiles dans la recherche ultérieure.

\section{L'apport linguistique}

Dans les langues occidentales, les désinences fournissent des précisions sur les relations grammaticales, fonctionnelles et logiques entre les éléments de la phrase (notamment par le biais des accords grammaticaux et des conjugaisons), et contribuent ainsi à délimiter le champ sémantique du terme inconnu.

En japonais, les désinences sont quasiment inexistantes, et le caractère elliptique et peu cartésien du langage ${ }^{10}$ offre peu de points d'appui pour l'analyse logicofonctionnelle de la phrase.

Par contre, des indices lexicaux précieux se présentent souvent. En effet, l'évolution de la terminologie scientifique et technique japonaise est quelque peu désordonnée, avec coxistence de synonymes multiples, dont certains sont des jiongo (mots formés avec des caractères chinois), d'autres des gairaigo (mots étrangers, généralement occidentaux, naturalisés japonais), d'autres encore des abréviations des jiongo $0^{11}$ ou des gairaigo (" wapu " pour "wado purasesa " - de "word processor", " machine de traitement de texte »). Certains de ces termes sont consacrés par des listes lexicographiques ou terminologiques, d'autres sont des créations personnelles. En parcourant le texte, on ren-

8. Voir B. Fujimori, op. cit., et Tadao Yokoi (1971) : Goyakuakuhaku no byori, Tokyo, Gendaijanarizmushuppansha, pp. 113-114, 138.

9. Voir par exemple le « Guide du traducteur » du ministère des Communications du Québec, Éditeur officiel du Québec, 1978, p. 7.

10. Évoqué par tous les auteurs japonais cités dans le présent article.

11. Voir Etsutaro Iwabuchi (1977) : Akubun, Tokyo, Nihonhyoronsha, 3e éd., p. 25. 
contre souvent des synonymes ou variantes du terme inconnu, qui sont plus accessibles en raison de leur morphologie, leur origine ou le contexte précis qui les entoure.

L'analyse contextuelle des textes japonais est donc essentiellement cognitive.

\section{LA RECHERCHE TERMINOLOGIQUE EXTERNE}

On le voit, l'analyse ponctuelle et l'analyse contextuelle ont essentiellement pour fonction l'élucidation du sens d'un terme inconnu. La recherche externe, pour sa part, est bivalente : elle aide à comprendre, mais aussi à réexprimer.

On peut distinguer la recherche terminologique " directe", reposant sur les lexiques, glossaires et dictionnaires, ainsi que sur les sources humaines ${ }^{12}$, et la recherche terminologique " indirecte", qui s'appuie sur des sources dont la vocation n'est pas lexicographique.

\section{Les dictionnaires dans la traduction japonais-français}

À propos de la recherche externe directe, on notera d'emblée une contrainte pratique dont les répercussions sont importantes : les dictionnaires spécialisés japonaisfrançais ou comprenant la combinaison japonais-français sont très peu nombreux. Cette situation, qui s'explique par la faible importance économique du français au Japon, a pour conséquence la nécessité, dans la recherche directe bilingue, de passer par une langue tierce, en général l'anglais.

Par ailleurs, les dictionnaires bilingues ou multilingues où figure le japonais sont publiés, dans leur quasi-totalité, par des maisons d'édition japonaises qui s'adressent à des utilisateurs japonais ; ils ont donc essentiellement pour mission d'expliquer à un lecteur japonais les termes inconnus qu'il pourrait rencontrer dans un texte en langue étrangère, plutôt que d'expliquer à un étranger les termes inconnus qu'il pourrait rencontrer dans un texte japonais. Toutes choses étant égales par ailleurs et compte tenu des contraintes générales mentionnées plus haut, le taux de complétude du vocabulaire retenu en japonais est inférieur au taux de complétude du vocabulaire retenu en langue de départ, et a fortiori, il se situe loin du maximum envisageable.

D'autre part, les auteurs de ces dictionnaires et leurs collaborateurs sont tous japonais (à quelques exceptions éventuelles près) ${ }^{13}$, ce qui s'explique par le petit nombre de spécialistes étrangers parlant japonais qui vivent au Japon ${ }^{14}$; la fiabilité linguistique de ces dictionnaires est donc pour le moins sujette à caution ${ }^{15}$.

Il existe cependant une catégorie de dictionnaires japonais dont la vocation n'est pas de donner des " équivalences ", mais d'expliquer des termes, ce qui est fait dans un court paragraphe, souvent illustré, où figure également, à titre accessoire, une désignation anglaise ${ }^{16}$. En tant que documents unilingues, ces dictionnaires sont généralement

12. Qui ne seront évoquées ici que marginalement.

13. À titre d'exemple, aucun nom étranger ne figure parmi les centaines de noms d'auteurs et de collaborateurs à la préparation de la dizaine de dictionnaires spécialisés qui ont été examinés spécialement aux fins du présent article.

14. Voir Tadashi Koretsune, "The Quality Control Concept in Technical Translation in Japan ", dans P. Horguelin (édit.) (1977) : "La traduction, une profession ", actes du VIIIe congrès de la FIT, Montréal, pp. $516-518$.

15. Voir Tadao Yokoi (1971) : Goyakuakuyaku no byori, Tokyo, Gebdauhabaruzumushuppankai, pp. 85, $91,104$.

16. Voir par exemple le Keizaishingojiten (économie), Tokyo, Nihonkeizaishimbunshahen, 1977, le Erekutoronikusuyogojiten (électronique), Tokyo, Dempashimbunsha, 1979, le Denkitsushinyogojiten (communications), Tokyo, Omsha, 1974, ou le Kompuyutayogojiten (informatique), Tokyo, Koronasha, 1978. 
de bonne qualité, et leur "bilinguisme » accessoire est souvent utile comme point de départ.

Les dictionnaires sont des outils imparfaits, jamais complets, jamais parfaitement fiables. Ce sont toutefois des outils précieux, grâce à leur accessibilité et à leur pertinence, qui en font souvent la seule voie d'accès à l'information recherchée, surtout en japonais, les autres types de sources terminologiques (documents divers, sources humaines) étant rares hors du Japon.

Il convient donc d'user des dictionnaires sans en abuser, en privilégiant les dictionnaires unilingues ${ }^{17}$ et en vérifiant dans la mesure du possible les informations recueillies à l'aide de "documents authentiques".

\section{LA RECHERCHE INDIRECTE}

Par "sources indirectes ", on entend ici les sources susceptibles de fournir des indications terminologiques sans en avoir la vocation. Il s'agit essentiellement de livres, d'articles et d'autres documents traitant du même sujet que le texte à traduire ou d'un sujet connexe.

L'exploitation de ces sources consiste à les examiner pour y rechercher des indications sur le sens d'un terme inconnu (surtout les sources en langue de départ) ou sur ses équivalents contextuels possibles (sources en langue d'arrivée); la démarche ressemble donc beaucoup à celle de l'analyse contextuelle.

Le temps d'accès à l'information dans la source indirecte est plus important que dans l'exploitation des sources directes, et la complétude peut être très faible. Par contre, les sources indirectes arrivent à un très fort degré de pertinence quand ce sont des documents du même type que le texte à traduire, et la fiabilité linguistique des documents authentiques (rédigés directement par des " locuteurs natifs ") est très supérieure à celle des sources directes.

Dans la traduction japonais-français en France (et de manière plus générale, hors du Japon), la recherche indirecte en langue de départ est handicapée par le manque de documents japonais. Par contre, l'absence de sources directes fait que les sources indirectes françaises sont très sollicitées en phase de restitution en langue d'arrivée.

\section{CONCLUSION}

La recherche terminologique dans la traduction scientifique et technique japonais-français en France est donc soumise à des contraintes particulières, qui en déplacent le centre de gravité par rapport à la recherche terminologique de type classique entre langues occidentales :

1. Plutôt que de s'appuyer sur la recherche externe directe en premier lieu, et sur la recherche contextuelle en deuxième lieu, elle se retrouve souvent cantonnée dans la recherche contextuelle et dans la recherche ponctuelle.

2. L'analyse ponctuelle y joue un rôle important.

3. Le traducteur utilise sensiblement plus de documents en langue d'arrivée qu'en langue de départ.

4. La recherche " directe" passe généralement par une langue tierce(!).

Ces contraintes font que dans la pratique professionnelle, le travail de recherche accompagnant la traduction japonais-français est dans l'ensemble plus long, plus ardu et plus analytique que le travail accompagnant la traduction entre langues occidentales. Sur le plan de la formation des traducteurs, cet état de choses appelle des méthodes particulières ${ }^{18}$.

17. Voir I. Pinchuk (1977) : Scientific and Technical Translation, London, André Deutsch, chap. 15.

18. Article en préparation. 
L'avènement des bases de données et de la télématique est susceptible de changer considérablement les données du problème à moyen ou à long terme, puisqu'il devrait permettre la mise en commun de ressources dispersées, le recueil d'un volume d'informations sans commune mesure avec la capacité maximale des sources externes classiques, et une mise à jour rapide et permanente.

Il se pourrait fort bien que d'ici dix ans, le travail de recherche terminologique du traducteur individuel indépendant soit profondément transformé par les progrès technologiques. Mais l'évolution touchera également, en toute probabilité, d'autres aspects de la profession, et c'est dans une perspective globale qu'il conviendra de revoir le problème.

DANIEL GILE 